Punjab University Journal of Mathematics

(ISSN 1016-2526)

Vol. 53(2)(2021) pp. 35-49

\title{
Generalized Topological Groupoids
}

\author{
Mustafa Habil Gürsoy \\ Department of Mathematics, \\ Faculty of Art and Science, \\ Inonu University, Turkey, \\ Email: mhgursoy@gmail.com
}

Received: 24 June, 2020 / Accepted: 09 February, 2021 / Published online: 22 Feburary, 2021

\begin{abstract}
Our aim in this paper is to give the notion of generalized topological groupoid which is a generalization of the topological groupoid by using the notion of generalized topology defined by Császár [6]. We investigate the basic facts in the groupoid theory in terms of generalized topological groupoids. We present the action of a generalized topological groupoid on a generalized topological space. We obtain some characterizations about this concept that is called the generalized topological action. Beside these, we give definition of a generalized topological crossed module by generalizing the concept of crossed module defined on topological groupoids. At the last part of the study, we show how a generalized topological crossed module can be obtained from a generalized topological groupoid and how a generalized topological groupoid can be obtained from a generalized topological crossed module.
\end{abstract}

AMS (MOS) Subject Classification Codes: 18B40; 18D35; 20L05; 22A22

Key Words: Generalized topology, generalized topological groupoid, generalized topological crossed module.

\section{INTRODUCTION}

After Császár [6] gave the notions of generalized topological space and generalized neighborhood systems in 2002, many topologists applied the concepts in general topology to the generalized topological spaces: separation axioms in generalized topologies, product of generalized topologies, generalized continuity, generalized compactness, generalized connectedness, etc. $[1,7,8,10,14,16]$

This topological concept was combined with algebra in 2013 by Hussain and et al. [12]. They have defined a generalized topological group as a group endowed with a generalized topology such that the multiplication and inversion of the group are generalized continuous according to this generalized topology. They have obtained some characterizations of the 
generalized topological group in their studies. Afterwards, in 2014, they defined the notion of generalized action using this concept [13].

Although the concept of groupoid which was the subject of this study was first defined as an algebraic concept by Brandt [2] in 1926, its categorical definition was given by Ehresmann [9] in 1958. In his own work, Ehresmann deals with the concept of action of a groupoid. Afterwards, the action of a groupoid has been studied from different perspectives by many mathematicians in terms of algebraic, topological and differentiable $[11,17]$.

Another concept we deal with in this study is the concept of crossed module. Crossed modules can be defined over several algebraic structures. The definition of crossed module over groupoids belongs to Brown and Higgins [4]. In this work, we use the definition given by Brown and Higgins.

In this study, we define the generalized topological groupoid by combining the concepts of generalized topological space and groupoid. We reinterpret the basic information in the groupoid theory using this new concept. We obtain the generalized topological action groupoid by defining the action of a generalized topological groupoid. Also, we present the concept of crossed module on the generalized topological groupoids which we called as generalized topological crossed module. Finally, we show how a generalized topological crossed module can be obtained from a generalized topological groupoid and how a generalized topological groupoid can be obtained from a generalized topological crossed module.

\section{PRELIMINARIES}

The basic reminders about the concepts to be used in the work will be here made. These basic reminders will be given in two subsections: $\mathcal{G}$-topological structures and groupoids.

\subsection{Generalized Topological Structures}

The concept of generalized topological space, defined by Császár, and some basic concepts to be used in study will be here recalled. In addition, some information about the generalized topological group defined by Hussain and et all will be given. For detailed information on these, readers may refer to [6] and [12].

Definition 2.1. [6] Let $\mathfrak{g}=\left\{O_{i} \mid O_{i} \subset M, i \in I\right\}$ denote a subfamily of power set $P(M)$, where $M$ is a nonempty set. If the emptyset $\emptyset$ belongs to $\mathfrak{g}$ and $\mathfrak{g}$ is closed under the arbitrary union, then $\mathfrak{g}$ is called a generalized topology. A pair $(M, \mathfrak{g})$ is also called generalized topological space or GTS.

Each $O_{i} \in \mathfrak{g}$ is said $\mathfrak{g}$-open set and the complement of $O_{i} \in \mathfrak{g}$ is said $\mathfrak{g}$-closed set. It is obvious that a $\mathfrak{g}$-closed set is complement of a $\mathfrak{g}$-open set. The union of all $O_{i} \in \mathfrak{g}$ is denoted by $\mathfrak{U}_{\mathfrak{g}}$. If $\mathfrak{U}_{\mathfrak{g}}=M$, then $(M, \mathfrak{g})$ is said to be strong. It is obvious that every topology is a generalized topology.

Example 2.2. For a set $M=\{m, n, k\}$, the subfamily

$$
\mathfrak{g}=\{\emptyset,\{m\},\{n\},\{m, n\}\} \subset P(M)
$$

is clearly a generalized topology.

Definition 2.3. [6] Let us consider the mapping $\xi: M \rightarrow P(P(M))$ and let us take any subset $V \in \xi(m)$ contains $m$ for any point $m \in M$. Then $V$ is called generalized 
neighborhood (G-neighborhood) of $m$. Also, for any $m \in M$ if $m \in V$ for all $V \in \xi(m)$, then $\xi$ is called a generalized neighbourhood system ( $\mathcal{G}$-neighborhood system) on $M$. We will denote the set of all $\mathcal{G}$-neighborhood systems on $M$ by $\Omega$ or $\Omega(M)$.

Now let us give a lemma by combining Lemma 1.2 and Lemma 1.3 of [6]. This lemma states that there is a match between generalized topologies on a set and generalized neighborhood systems. More precisely, a generalized neighborhood system determines some generalized topology, and conversely a generalized topology determines some generalized neighborhood systems.

Lemma 2.4. [12] Let $\Omega$ be a $\mathcal{G}$-neighborhood system on $M$, let $\mathfrak{g} \subset P(M)$. For $O \in \mathfrak{g}$ and $m \in O$, there exists a subset $U \in \xi(m), \xi \in \Omega, m \in U \subset O$ if and only if $\mathfrak{g}$ is $a$ $\mathcal{G}$-topology on $M$.

Definition 2.5. [6] A map $\rho:(M, \mathfrak{g}) \rightarrow(N, \mathfrak{h})$ is said

i) $(\mathfrak{g}, \mathfrak{h})$-continuous if $\rho^{-1}(O) \in \mathfrak{g}$ for any $O \in \mathfrak{h}$.

ii) $(\mathfrak{g}, \mathfrak{h})$-open if $\rho(O) \in \mathfrak{h}$ for any $O \in \mathfrak{g}$.

iii) $(\mathfrak{g}, \mathfrak{h})$-homeomorphism if $\rho$ is bijective, $(\mathfrak{g}, \mathfrak{h})$-continuous, and $\rho^{-1}$ is $(\mathfrak{h}, \mathfrak{g})$-continuous.

Throughout the paper we will use the notations $\mathcal{G}$-continuous, $\mathcal{G}$-open, $\mathcal{G}$-homeomorphism as independent of the generalized topologies for the concepts above.

Definition 2.6. [10] Let $(M, \mathfrak{g})$ be a GTS and $\emptyset \neq N \subset M$. Then the subspace $\mathcal{G}$-topology of $N$ is $\mathcal{G}$-topology $\mathfrak{g}_{N}=\{N \cap O \mid O \in \mathfrak{g}\}$ on $N$. The pair $\left(N, \mathfrak{g}_{N}\right)$ is called a subspace $\operatorname{GTS}$ of $(M, \mathfrak{g})$.

Lemma 2.7. [10] If $\rho:(M, \mathfrak{g}) \rightarrow(N, \mathfrak{h})$ is $\mathcal{G}$-continuous, then for any $\emptyset \neq K \subset M$ the restriction $\left.\rho\right|_{K}:\left(K, \mathfrak{g}_{K}\right) \rightarrow\left(\rho(K), \mathfrak{h}_{\rho(K)}\right)$ is $\mathcal{G}$-continuous.

Let us now give the definition of $\mathcal{G}$-topological group.

Definition 2.8. [12] A $\mathcal{G}$-topological group $\mathbb{G}$ is a group endowed with a $\mathcal{G}$-topology such that the structure maps of $\mathbb{G}$ are $\mathcal{G}$-continuous maps.

Example 2.9. [12] The group $(I R,+)$ is a GTS under the generalized topology $\mathfrak{g}$ generated by the basis $\mathcal{A}=\left\{\left(-\infty, a_{1}\right),\left(a_{2}, \infty\right): a_{1}, a_{2} \in I R\right\}$, where IR denotes the real numbers. Therefore, $((I R,+), \mathfrak{g})$ is a $\mathcal{G}$-topological group.

Proposition 2.10. [12] Any subgroup of a $\mathcal{G}$-topological group is also a $\mathcal{G}$-topological group. It is called $\mathcal{G}$-topological subgroup.

Definition 2.11. [12] Let $(\mathbb{G}, \mathfrak{g})$ and $\left(\mathbb{G}^{\prime}, \mathfrak{g}^{\prime}\right)$ be two $\mathcal{G}$-topological groups and $\tau: \mathbb{G} \rightarrow \mathbb{G}^{\prime}$ be a group homomorphism. If $\tau$ is $\mathcal{G}$-continuous, it is called a $\mathcal{G}$-homomorphism.

If $\tau$ is a $\mathcal{G}$-homeomorphism, then it is said a $\mathcal{G}$-isomorphism. The composition of two $\mathcal{G}$ homomorphisms is also a $\mathcal{G}$-homomorphism. Further, the identity map is a $\mathcal{G}$-isomorphism. Thus, $\mathcal{G}$-topological groups form a category denoted by $\mathcal{G T} G$.

Definition 2.12. [12] Let $(M, \mathfrak{g})$ be a GTS. If for any $m_{1}, m_{2} \in M$ there is a $\mathcal{G}$ - homeomorphism $\rho:(M, \mathfrak{g}) \rightarrow(M, \mathfrak{g})$ satisfying $\rho\left(m_{1}\right)=m_{2}$, then $M$ is called $\mathcal{G}$-homogeneous.

Theorem 2.13. [12] Let $(\mathbb{G}, \mathfrak{g})$ be a $\mathcal{G}$-topological group. For any $a \in \mathbb{G}$, the left translation $l_{a}:(\mathbb{G}, \mathfrak{g}) \rightarrow(\mathbb{G}, \mathfrak{g}), l_{a}(x)=$ ax is a $\mathcal{G}$-homeomorphism. 
This theorem is also valid to the right translation.

Each $\mathcal{G}$-topological group $(\mathbb{G}, \mathfrak{g})$ is $\mathcal{G}$-homogeneous, namely for any elements $a, a^{\prime} \in \mathbb{G}$ there is always a $\mathcal{G}$-homeomorphism $l_{a^{\prime} a^{-1}}:(\mathbb{G}, \mathfrak{g}) \rightarrow(\mathbb{G}, \mathfrak{g})$ satisfying $l_{a^{\prime} a^{-1}}(a)=a^{\prime}$.

Theorem 2.14. [12] A $\mathcal{G}$-homomorphism which is $\mathcal{G}$-continuous at the identity is $\mathcal{G}$ - continuous everywhere.

Theorem 2.15. [12] If a subgroup $\mathbb{H}$ of a $\mathcal{G}$-topological group $\mathbb{G}$ contains a non-empty $\mathcal{G}$-open set, then it is $\mathcal{G}$-open in $\mathbb{G}$.

For the following proposition, let us recall the definition of a Hausdorff GTS from [8].

Definition 2.16. [8] Let $(M, \mathfrak{g})$ be a GTS. If for each pair of points $m_{1} \neq m_{2}$, there exist $\mathcal{G}$-open sets $O_{1}, O_{2} \in \mathfrak{g}$ satisfying $m_{1} \in O_{1}$ and $m_{2} \in O_{2}$ such that $O_{1} \cap O_{2}=\emptyset$, then $(M, \mathfrak{g})$ is called Hausdorff GTS.

Proposition 2.17. [12] In a Hausdorff $G T S(M, \mathfrak{g})$, each singleton is a $\mathcal{G}$-closed.

By the proposition above, for a $\mathcal{G}$-topological group $\mathbb{G}$, the set $\{e\}$ is $\mathcal{G}$-closed, where $e$ is the identity of $\mathbb{G}$.

Definition 2.18. [13] Let $\mathbb{G}$ be a $\mathcal{G}$-topological group and let $M$ be a GTS. A $\mathcal{G}$-action of $\mathbb{G}$ on $M$ is a $\mathcal{G}$-continuous map $: \mathbb{G} \times M \rightarrow M$ such that $e^{\cdot} m=m$ and $(g h)^{\cdot} m=g^{\cdot}(h m)$, for e, $g, h \in \mathbb{G}, m \in M$.

The space $M$ is said $\mathcal{G}$ - $\mathbb{G}$-space. For any $m \in M$, the set

$$
\mathbb{G}(m)=\{n \mid n=g \cdot m, \exists g \in \mathbb{G}\}
$$

is called the orbit of $m$.

Proposition 2.19. [13] Every $\mathcal{G}$-action $\sigma: \mathbb{G} \times M \rightarrow M$ is a $\mathcal{G}$-open map.

Proof. For any $\mathcal{G}$-open sets $O$ and $V$ in $\mathbb{G}$ and $M$, resp., let us take $O \times V \subset \mathbb{G} \times M$. Then, $\sigma(O \times V)=\bigcup_{g \in \mathbb{G}} \sigma_{g}(V)$ is a $\mathcal{G}$-open set, because each map $\sigma_{g}: M \rightarrow M$ is a $\mathcal{G}$-homeomorphism. Since the $\mathcal{G}$-open sets $O \times V$ form a basis of $\mathbb{G} \times M$, the map $\sigma$ is $\mathcal{G}$-open.

Example 2.20. [13] The action of a $\mathcal{G}$-topological group $\mathbb{G}$ on itself by the left translation is a $\mathcal{G}$-action. Because, the action has been defined by the multiplication of $\mathbb{G}$, i.e., $\sigma(g, h)=$ $g h, \forall g, h \in \mathbb{G}$.

Definition 2.21. [13] Let $\mathbb{G}$ be a $\mathcal{G}$-topological group, let $M, N$ be two GTSs, let $\sigma_{M}$ : $\mathbb{G} \times M \rightarrow M$ and $\sigma_{N}: \mathbb{G} \times N \rightarrow N$ be two $\mathcal{G}$-actions. Then, a $\mathcal{G}$-continuous map $\vartheta: M \rightarrow N$ is said $\mathbb{G}$-equivariant if the diagram

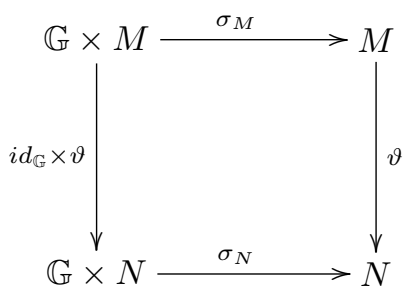


is commutative, namely, $\vartheta\left(\sigma_{M}(g, m)\right)=\sigma_{N}(g, \vartheta(m)), \forall g \in \mathbb{G}, \forall m \in M$.

Definition 2.22. [13] Let $M$ be a $\mathcal{G}$-G্-space. Let us take any element $m \in M$. The stabilizer of $m$ in $\mathbb{G}$ is the set $\mathbb{G}_{m}=\left\{a_{1} \in \mathbb{G} \mid a_{1} m=m\right\}$ which is a subgroup of $\mathbb{G}$.

It is clear that if $\mathbb{G}$ is Hausdorff, then the singleton $\{m\}$ is $\mathcal{G}$-closed. Hence, $\mathbb{G}_{m}$ is $\mathcal{G}$-closed, so $\mathbb{G}_{m}$ is a $\mathcal{G}$-topological group.

Let $M$ be a $\mathcal{G}$ - $\mathbb{G}$-space. Then, for $m \in M$ we have a map $\mu_{m}: \mathbb{G} \rightarrow M, \mu_{m}(g)=g^{\prime} m$ which is $\mathcal{G}$-continuous. Hence, we have

Definition 2.23. [13]

i) If $\mu_{m}$ is surjective, the $\mathcal{G}$-action is called transitive.

ii) If $\bigcap_{m \in M} \mathbb{G}_{m}=\{e\}$, the $\mathcal{G}$-action is called effective.

\subsection{Groupoids}

In this subsection, we give some basic reminders about the concept of groupoid which is the subject of the study. See [3] for details on groupoids.

Definition 2.24. [3] A groupoid consists of the set $\Gamma$ of the arrows and the set $\Gamma_{0}$ of the objects, together with the source map $s: \Gamma \rightarrow \Gamma_{0}$, target map $t: \Gamma \rightarrow \Gamma_{0}$, the object map $\epsilon: \Gamma_{0} \rightarrow \Gamma, m \mapsto \epsilon(m)=1_{m}$, the inverse map inv $: \Gamma \rightarrow \Gamma, \alpha \mapsto$ inv $(\alpha)=\alpha^{-1}$, and $a$ partial multiplication $(\alpha, \beta) \mapsto \alpha \circ \beta$ defined on the pullback $\Gamma_{2}=\Gamma_{s} \times_{t} \Gamma=\{(\alpha, \beta) \mid$ $s(\alpha)=t(\beta)\}$.

We denote a groupoid $\Gamma$ over $\Gamma_{0}$ by $\left(\Gamma, \Gamma_{0}\right)$ or $\Gamma$ or $\left(\Gamma, s, t, \Gamma_{0}\right)$. The maps in the definition above are said structure maps of groupoid. For a groupoid $\Gamma$ over $\Gamma_{0}$ and $m, n \in$ $\Gamma_{0}$, we have the sets $S t_{\Gamma} m=s^{-1}(m), \operatorname{CoSt}_{\Gamma} n=t^{-1}(n)$ and $S t_{\Gamma} m \cap \operatorname{CoSt}_{\Gamma} n=$ $\Gamma(m, n)$. Also, the set $\Gamma(m, m)$ (or only $\Gamma(m))$ is a group with the partial multiplication in $\Gamma$. We say it vertex group at $m$. A groupoid $\Gamma$ is called transitive if $\Gamma(m, n) \neq \emptyset$ for all $m, n \in \Gamma_{0}$. A groupoid $\Gamma$ is called totally intransitive if $\Gamma(m, n)=\emptyset$ for every $m, n \in \Gamma_{0}$ satisfying $m \neq n$.

Example 2.25. [3]

i) Every group is a groupoid over its identity.

ii) Every set $M(\neq \emptyset)$ can be thought as a groupoid over itself with $s=t=i d_{M}$

iii) For a set $M(\neq \emptyset)$, the product $M \times M$ is a groupoid over $M$. The arrows are ordered pairs $(m, n), m, n \in M$.

Let us give an example that will be used in the next section.

Example 2.26. [3] Let $M$ be a set, $\Omega$ a group and let an action of $\Omega$ on $M$ be $: \Omega \times M \rightarrow$ $M,(\alpha, m) \mapsto \alpha m$. We consider the pair of $(\alpha, m)$ as an arrow whose the source is $m$ and the target is $\alpha \cdot m$. The partial multiplication is defined by $(\beta, n) \circ(\alpha, m)=(\beta \alpha, m)$, where $n=\alpha \cdot m$. The partial multiplication defined in this way is well-defined. According to this operation, the unit arrow in $m \in M$ is $(e, m)$ and the inverse of $(\alpha, m)$ is $\left(\alpha^{-1}, \alpha^{\prime} m\right)$. The groupoid $\Omega \times M$ constructed in this way is called "action groupoid".

Definition 2.27. $[3,11]$ A groupoid homomorphism from $\left(\Gamma, \Gamma_{0}\right)$ to $\left(\Phi, \Phi_{0}\right)$ is a pair $\left(\lambda, \lambda_{0}\right)$ of maps $\lambda: \Gamma \rightarrow \Phi, \lambda_{0}: \Gamma_{0} \rightarrow \Phi_{0}$ such that $s_{\Phi} \circ \lambda=\lambda_{0} \circ s_{\Gamma}, t_{\Phi} \circ \lambda=\lambda_{0} \circ t_{\Gamma}$ and $\lambda(\alpha \circ \beta)=\lambda(\alpha) \circ \lambda(\beta), \forall(\alpha, \beta) \in \Gamma_{2}$. 
We sometimes use the only map $\lambda$ for $\left(\lambda, \lambda_{0}\right)$. If $\lambda$ is a bijection, then $\lambda: \Gamma \rightarrow \Phi$ is a groupoid isomorphism.

Thus, it is obtained the category $G p d$ of the groupoids and groupoid homomorphisms.

A subgroupoid $\left(\Gamma^{*}, \Gamma_{0}^{*}\right)$ of $\left(\Gamma, \Gamma_{0}\right)$ is a groupoid with $\Gamma^{*} \subset \Gamma, \Gamma_{0}^{*} \subset \Gamma_{0}$, having the same source and target maps and partial multiplication. A subgroupoid $\Gamma^{*}$ is called full if $\Gamma^{*}(m, n)=\Gamma(m, n)$ for all $m, n \in \Gamma_{0}^{*}$, and is called wide if $\Gamma_{0}^{*}=\Gamma_{0}$.

Let $\Delta$ be a wide subgroupoid of a groupoid $\Gamma$. For all $m, n \in \Gamma_{0}$ and $\alpha \in \Gamma(m, n)$, if we have $\alpha \Delta(m)=\Delta(n) \alpha$, then $\Delta$ is called a normal subgroupoid of $\Gamma$, where $\Delta(m)$ and $\Delta(n)$ denote vertex groups at $m$ and $n$, respectively.

Example 2.28. [3] For a groupoid homomorphism $\lambda: \Gamma \rightarrow \Phi$, the kernel ker $\lambda=\{\alpha \in$ $\left.\Gamma \mid \lambda(\alpha)=1_{m}, \exists m \in \Phi_{0}\right\}$ is a normal subgroupoid of $\Gamma$, where $1_{m}: m \rightarrow m$ is the identity arrow of $\left(\Phi, \Phi_{0}\right)$.

Definition 2.29. [3] In a groupoid $\left(\Gamma, \Gamma_{0}\right)$, if $\Gamma$ is a topological space and structure maps are continuous, then it is called a topological groupoid.

It is clear that a homomorphism between topological groupoids $\left(\Gamma, \Gamma_{0}\right)$ and $\left(\Phi, \Phi_{0}\right)$ is a groupoid homomorphism $\left(\lambda, \lambda_{0}\right):\left(\Gamma, \Gamma_{0}\right) \rightarrow\left(\Phi, \Phi_{0}\right)$ such that $\lambda$ and $\lambda_{0}$ are continuous.

Let us express the groupoid action.

Definition 2.30. [11, 17] Let $\left(\Gamma, \Gamma_{0}\right)$ be a groupoid and let $M$ be a set. Let $J: M \rightarrow \Gamma_{0}$ be a map. A left action of $\Gamma$ on $M$ via $J$ is a map $\phi: \Gamma_{s \times} M \rightarrow M,(\alpha, m) \mapsto \alpha \cdot m$, is called moment map, satisfying the conditions
i) $J(\alpha \cdot m)=t(\alpha)$
ii) $\beta \cdot(\alpha \cdot x)=(\beta \circ \alpha) \cdot m$
iii) $\left(1_{J(m)}\right) \cdot m=m$,

for any $\alpha, \beta \in \Gamma, m \in M$. The set $M$ is said a left $\Gamma$-set.

A right action of $\Gamma$ on $M$ is also defined similarly. In this case, we have the map $\phi: M_{J^{\times}} \Gamma \rightarrow M,(m, \alpha) \mapsto m \cdot \alpha$ and the first condition becomes $J(m \cdot \alpha)=s(\alpha)$.

Example 2.31. [11, 17]

(1) Any groupoid $\Gamma$ acts on itself from both sides by the partial multiplication of $\Gamma$. The moments are $s$ and $t$ for the left and right actions, respectively.

(2) Any groupoid $\Gamma$ acts on $\Gamma_{0}$ from both sides with moment $i d_{\Gamma_{0}}$. The left action is $\alpha m=$ $t(\alpha)$ and the right action is $m^{\circ} \alpha=s(\alpha)$.

Definition 2.32. [4] A crossed module over groupoids is a triplet $\mathcal{C}=(\Upsilon, \Gamma, \eta)$ together with a (left) action of $\Gamma$ on $\Upsilon$ and a functor $\eta: \Upsilon \rightarrow \Gamma$, where the groupoids $\Upsilon$ and $\Gamma$ have the same object space and $\Upsilon$ is totally intransitive, such that the following conditions hold: CR1) $\eta(\alpha \omega)=\alpha \circ \eta(\omega) \circ \alpha^{-1}$ for $\omega \in \Upsilon(m, m), \alpha \in \Gamma(m, n)$

CR2) $\eta\left(\omega_{1}\right)^{\cdot} \omega=\omega_{1} \circ \omega \circ \omega_{1}^{-1}$ for $\omega, \omega_{1} \in \Upsilon(m, m)$.

Example 2.33. [5] Every groupoid $\Gamma$ over $\Gamma_{0}$ constructs a crossed module over groupoids with its inner group $I \Gamma=\bigcup \Gamma(m)$. The inclusion inc $: I \Gamma \rightarrow \Gamma$ is a groupoid homomorphism, and the groupoid $\Gamma$ acts as follows on $I \Gamma$ from left.

$$
\begin{aligned}
& : \Gamma \times I \Gamma \rightarrow I \Gamma \\
& (\alpha, \omega) \mapsto \alpha^{\prime} \omega=\alpha \circ \omega \circ \alpha^{-1}
\end{aligned}
$$

Therefore, the triplet $(I \Gamma, \Gamma$, inc) is a crossed module over groupoids. 
Definition 2.34. [5] Let $\left(\Upsilon_{1}, \Gamma_{1}, \eta_{1}\right)$ and $\left(\Upsilon_{2}, \Gamma_{2}, \eta_{2}\right)$ be crossed modules. A crossed module homomorphism $(\tau, \psi):\left(\Upsilon_{1}, \Gamma_{1}, \eta_{1}\right) \rightarrow\left(\Upsilon_{2}, \Gamma_{2}, \eta_{2}\right)$ is a pair of groupoid homomorphisms $\tau: \Upsilon_{1} \rightarrow \Upsilon_{2}$ and $\psi: \Gamma_{1} \rightarrow \Gamma_{2}$ such that the following equalities are hold:

i) $\eta_{2}(\tau(\omega))=\psi\left(\eta_{1}(\omega)\right)$, for all $\omega \in \Upsilon_{1}$

ii) $\tau\left(\alpha^{\cdot} \omega\right)=\psi(\alpha)^{\cdot} \tau(\omega)$, for all $\omega \in \Upsilon_{1}, \alpha \in \Gamma_{1}$.

Therefore, we construct the category $\operatorname{Crs} M$ of the crossed modules over groupoids.

\section{3. $\mathcal{G}$-TopologicAl GROUPOIDS}

We here define the generalized topological groupoid that is a generalization of topological groupoids by combining the concepts of generalized topological space and groupoid. We examine some basic information in the groupoid theory in terms of generalized topological groupoids. Also we obtain some characterizations about it by defining the action of a generalized topological groupoid on a GTS.

Definition 3.1. A generalized topological groupoid is a groupoid $\Gamma$ endowed with a $\mathcal{G}$ topology $\mathfrak{g}$ such that all structure maps of $\Gamma$ are $\mathcal{G}$-continuous.

We will write $\mathcal{G}$-topological groupoid for a generalized topological groupoid, for brevity.

Example 3.2. Given any $G T S(M, \mathfrak{g})$, if we take $\Gamma_{0}=\Gamma=M$ and as identity map all structure maps, one can obtain a $\mathcal{G}$-topological groupoid.

Example 3.3. Given any Haussdorf $\mathcal{G}$-topological group $\mathbb{G}$, if it is taken as $\Gamma_{0}=\{\star\}$, $s, t: \Gamma=\mathbb{G} \rightarrow\{\star\}, \epsilon(\star)=e_{\mathbb{G}}$, one can obtain a $\mathcal{G}$-topological groupoid $\Gamma$ over the singleton $\{\star\}$ with the operations of $\mathbb{G}$.

Example 3.4. For any strong GTS $(M, \mathfrak{g})$, the cartesian product $M \times M$ is viewed as $\mathcal{G}$-topological groupoid over $(M \times M)_{0}=M$. The source and target maps are the first and second projection, respectively. By Proposition. 2.7 in [7], they are $\mathcal{G}$-continuous maps. The object map $\epsilon: M \rightarrow M \times M$ defined by $\epsilon(m)=(m, m)$ is $\mathcal{G}$-continuous, since $\epsilon^{-1}(O \times O)=O$ is clearly $\mathcal{G}$-open, where $m \in O$ and $O \in \mathfrak{g}$. The multiplication is both unique and $\mathcal{G}$-continuous, because for any $m, n \in M$ there is only one arrow from $m$ to $n$. The inverse map inv : $M \times M \rightarrow M \times M$, inv $(m, n)=(n, m)$ is $\mathcal{G}$-continuous. Because, for each $\mathcal{G}$-open set $O_{2} \times O_{1}$ containing $(n, m)$, the set inv ${ }^{-1}\left(O_{2} \times O_{1}\right)=O_{1} \times O_{2}$ is $\mathcal{G}$-open set containing $(m, n)$ in $M \times M$.

Example 3.5. For a strong $\mathcal{G}$-topological group $\Gamma$ and a strong $G T S M$, let us consider $\mathcal{G}$-action of $\Gamma$ on $M$. Then we construct a $\mathcal{G}$-topological groupoid $\Gamma \ltimes M$ over $M$, called action $\mathcal{G}$-topological groupoid, as follows:

$$
\begin{gathered}
s(\alpha, m)=m, t(\alpha, m)=\alpha \cdot m, \varepsilon(m)=(e, m), \operatorname{inv}(\alpha, m)=\left(\alpha^{-1}, \alpha \cdot m\right) \\
(\beta, n) \circ(\alpha, m)=(\beta \alpha, m), \text { whenever } n=\alpha \cdot m .
\end{gathered}
$$

The space of objects is clearly a GTS. From Proposition.2.7 in [7], the space of arrows has a structure of $\mathcal{G}$-topological space. Since the source map is the first projection, it is $\mathcal{G}$-continuous map. The target map is $\mathcal{G}$-continuous, because it is defined by the $\mathcal{G}$-action. Let us show that the object map is $\mathcal{G}$-continuous. First of all, let us state that $\Gamma \ltimes M$ has a 
product $\mathcal{G}$-subspace structure reduced from $\Gamma \times M$. Now,let $O$ and $U$ be $\mathcal{G}$-open sets in $\Gamma$ and $M$, respectively. Then, for any $\mathcal{G}$-open set $O \times U$, we have

$$
\varepsilon^{-1}(O \times U)= \begin{cases}U & , \text { if } e \in O \\ \emptyset & , \text { if } e \notin O .\end{cases}
$$

From here it is easily seen that $\varepsilon^{-1}(O \times U)$ is $\mathcal{G}$-open in $\Gamma$. So, the object map $\varepsilon$ is $\mathcal{G}$ continuous. The inverse map inv is $\mathcal{G}$-continuous, because it is the product of the inversion in $\Gamma$ and the $\mathcal{G}$-action. Since they are $\mathcal{G}$-continuous, the map inv is also $\mathcal{G}$-continuous. $F i$ nally, let us show that the partial multiplication is $\mathcal{G}$-continuous. The partial multiplication can be written as a composition of maps as shown in the diagram below.

$$
\begin{gathered}
(\Gamma \ltimes M)_{s^{\times} t}(\Gamma \ltimes M) \stackrel{C_{2,3}}{\longrightarrow}(\Gamma \times \Gamma) \times(M \times M) \stackrel{\mu \times I d}{\longrightarrow} \Gamma \times M \times M \stackrel{\left(\pi_{1} \times \pi_{3}\right)}{\longrightarrow}(\Gamma \ltimes M) \\
((\beta, n),(\alpha, m)) \longmapsto((\beta, \alpha),(n, m)) \longmapsto(\beta \alpha, n, m) \longmapsto(\beta \alpha, m)
\end{gathered}
$$

, where $\mu$ is the composition of $\mathcal{G}$-topological group $\Gamma, \pi_{1}$ is the first projection, $\pi_{3}$ is the third projection and $C_{2,3}$ is the twisting function that changes the locations of the second and third elements. Since all of these maps are $\mathcal{G}$-continuous, the partial multiplication is $\mathcal{G}$-continuous.

Therefore, $\Gamma \ltimes M$ is a $\mathcal{G}$-topological groupoid.

Definition 3.6. A $\mathcal{G}$-topological groupoid homomorphism from $\left(\Gamma, \Gamma_{0}\right)$ to $\left(\Gamma^{\prime}, \Gamma_{0}^{\prime}\right)$ is a groupoid homomorphism $\left(\lambda, \lambda_{0}\right)$ such that $\lambda$ and $\lambda_{0}$ are $\mathcal{G}$-continuous maps.

This yields the category $G T G p d$ of $\mathcal{G}$-topological groupoids and their homomorphisms.

Example 3.7. Let $\left(\Gamma, s, t, \Gamma_{0}\right)$ be a topological groupoid. Since every topological space is a GTS, $\left(\Gamma, s, t, \Gamma_{0}\right)$ is also a $\mathcal{G}$-topological groupoid. Let us consider the $\mathcal{G}$-topological groupoid $\left(\Gamma_{0} \times \Gamma_{0}, \widetilde{s}, \widetilde{t}, \triangle_{\Gamma_{0}}\right)$ associated to $\Gamma_{0}$. Then the anchor map $(s, t): \Gamma \rightarrow \Gamma_{0} \times \Gamma_{0}$ is a homomorphism of $\mathcal{G}$-topological groupoids. Indeed, the generalized continuity of the anchor map $(s, t)$ follows from the generalized continuity of $s$ and $t$. Namely, let us consider $\mathcal{G}$-open sets $O$ and $U$ in $\mathfrak{g}_{\Gamma_{0}}$ of $s(\alpha) \in \Gamma_{0}$ and $t(\alpha) \in \Gamma_{0}$, resp., for any arrow $\alpha \in \Gamma$. Then $O \times U$ is a basic $\mathcal{G}$-open set containing $(s(\alpha), t(\alpha))$. Since $\left(\Gamma, s, t, \Gamma_{0}\right)$ is a topological groupoid, the set $(s, t)^{-1}(O \times U)=s^{-1}(O) \cap t^{-1}(U)$ is $\mathcal{G}$-open set in $\Gamma$. So, the anchor map $(s, t)$ is $\mathcal{G}$-continuous. On the other hand, from the groupoid theory, the anchor map is a groupoid homomorphism. Thus $(s, t): \Gamma \rightarrow \Gamma_{0} \times \Gamma_{0}$ is a homomorphism of $\mathcal{G}$-topological groupoids.

Definition 3.8. Let $\Gamma$ be a $\mathcal{G}$-topological groupoid over $\Gamma_{0}$. A $\mathcal{G}$-subgroupoid of $\Gamma$ is a pair of $\left(\Gamma^{*}, \Gamma_{0}^{*}\right)$ of $\mathcal{G}$-subspaces $\Gamma^{*} \subset \Gamma, \Gamma_{0}^{*} \subset \Gamma_{0}$ together with the restriction structure maps. $A$ $\mathcal{G}$-subgroupoid $\left(\Gamma^{*}, \Gamma_{0}^{*}\right)$ is called wide if $\Gamma_{0}^{*}=\Gamma_{0}$, and is called full if $\Gamma^{*}(m, n)=\Gamma(m, n)$ for all $m, n \in \Gamma_{0}^{*}$.

The identity $\mathcal{G}$-subgroupoid of a $\mathcal{G}$-topological groupoid $\Gamma$ is the subgroupoid $\epsilon\left(\Gamma_{0}\right)=$ $\left\{1_{m} \mid m \in \Gamma_{0}\right\}$ with a subspace $\mathcal{G}$-topology induced from $\Gamma$. The inner $\mathcal{G}$-subgroupoid of $\Gamma$ is the subgroupoid $I \Gamma=\bigcup_{m \in \Gamma_{0}} \Gamma(m, m)$. 
Definition 3.9. A normal $\mathcal{G}$-subgroupoid of a $\mathcal{G}$-topological groupoid $\left(\Gamma, \Gamma_{0}\right)$ is a wide $\mathcal{G}$ subgroupoid $\Delta$ such that for any $\alpha \in \Delta(m, m)$ and any $\beta \in \Gamma$ with $t(\beta)=s(\alpha)=t(\alpha)$, we have $\beta \alpha \beta^{-1} \in \Delta$.

Definition 3.10. The kernel of a $\mathcal{G}$-homomorphism $\left(\lambda, \lambda_{0}\right):\left(\Gamma, \Gamma_{0}\right) \rightarrow\left(\Gamma^{\prime}, \Gamma_{0}^{\prime}\right)$ is the set $\operatorname{ker} \lambda=\left\{\alpha \in \Gamma \mid \lambda(\alpha)=1_{m}\right.$ for some $\left.m \in \Gamma_{0}^{\prime}\right\}$.

Example 3.11. Let $\lambda: \Gamma \rightarrow \Gamma^{\prime}$ be a $\mathcal{G}$-homomorphism. Then, from Definition 2.6, ker $\lambda$ has clearly the structure of $\mathcal{G}$-subspace. Also ker $\lambda$ is the wide $\mathcal{G}$-subgroupoid of $\Gamma$, and so it is a normal $\mathcal{G}$-subgroupoid of $\Gamma$. It is easily seen that ker $\lambda$ is wide in $\Gamma$, and normality follows from

$$
\lambda\left(\alpha \gamma \alpha^{-1}\right)=\lambda(\alpha) \lambda(\gamma) \lambda\left(\alpha^{-1}\right)=\lambda(\alpha) \lambda\left(\alpha^{-1}\right)=1, \gamma \in \operatorname{ker} \lambda(m), \alpha \in \Gamma(m, n) .
$$

Definition 3.12. Let $\left(\Gamma, \Gamma_{0}\right)$ be a $\mathcal{G}$-topological groupoid. $\Gamma$ is transitive if its underlying groupoid is transitive, and is totally intransitive if its underlying groupoid is totally intransitive.

As an example, it is obvious that the identity $\mathcal{G}$-subgroupoid and the inner $\mathcal{G}$-subgroupoid of $\Gamma$ are totally intransitive, because their underlying groupoids are totally intransitive.

Let us now give action of a $\mathcal{G}$-topological groupoid on a GTS.

Definition 3.13. Let $\Gamma$ be a $\mathcal{G}$-topological groupoid and let $M$ be a GTS. Let $J: M \rightarrow \Gamma_{0}$ be a $\mathcal{G}$-continuous map, called moment map. A left $\mathcal{G}$-action of $\Gamma$ on $M$ via $J$ is a $\mathcal{G}$ continuous map $\phi: \Gamma_{s \times J} M \rightarrow M,(\alpha, m) \mapsto \phi(\alpha, m)=\alpha \cdot m$ verifying the equations i) $J(\phi(\alpha, m))=t(\alpha)$, ii $) \phi(\beta,(\phi(\alpha, m))=\phi((\beta \circ \alpha), m)$, iii $) \phi\left(1_{J(m)}, m\right)=m$, where $\alpha, \beta \in \Gamma, m \in M$. The set $M$ is called a left $\mathcal{G}$ - $\Gamma$-space. Similarly, it can be also define a right $\mathcal{G}$-action.

Example 3.14. Every $\mathcal{G}$-topological groupoid $\Gamma$ acts on itself from both sides by the partial multiplication of $\Gamma$. The moment maps are $s$ and $t$ for the left and right $\mathcal{G}$-actions, respectively.

Example 3.15. Let $\left(\Gamma, s, t, \Gamma_{0}\right)$ be a $\mathcal{G}$-topological groupoid. Then $\Gamma$ acts on the $M=\Gamma_{0}$ via the $\mathcal{G}$-continuous map $J=I d: M=\Gamma_{0} \rightarrow \Gamma_{0}$. It is easily shown that the conditions of $\mathcal{G}$-action are hold. So, it is enough to say that the action map

$$
\phi: \Gamma_{s} \times_{J} \Gamma_{0} \rightarrow \Gamma_{0},(\alpha, m) \mapsto \phi(\alpha, m)=t(\alpha) .
$$

is $\mathcal{G}$-continuous. Clearly, since $t$ is $\mathcal{G}$-continuous, the action $\phi$ is $\mathcal{G}$-continuous.

Let $\Gamma$ be a $\mathcal{G}$-topological groupoid over $\Gamma_{0}$, and $M$ a $\mathcal{G}$ - $\Gamma$-space. For $x \in \Gamma_{0}$, we call the $\Gamma_{x}=s^{-1}(x) \cap t^{-1}(x)$ a $\mathcal{G}$-stable subgroup. Namely, $\Gamma_{x}$ is a $\mathcal{G}$-topological group with the structure of $\mathcal{G}$-subspace induced from $\mathcal{G}$-topological groupoid $\Gamma$. Also, the multiplication of $\Gamma_{x}$ is partial multiplication in $\Gamma$. The unit element is $x$ and every element $\alpha \in \Gamma_{x}$ has an inverse element $\alpha^{-1}$.

The action $\mathcal{G}$-topological groupoid given in Example 3.5 was constructed using the $\mathcal{G}$-topological group action. Now let's give its construction in case of the $\mathcal{G}$-topological groupoid action.

Example 3.16. Let $\left(\Gamma, s, t, \mu, \epsilon, i n v, \Gamma_{0}\right)$ be a $\mathcal{G}$-topological groupoid acting on GTS $M$ via $J: M \rightarrow \Gamma_{0}$. Then we can obtain a $\mathcal{G}$-topological groupoid with the space of objects 
that is the GTS $M$ and the space of morphisms is GTS $\Gamma_{s} \times_{J} M$. It is called action $\mathcal{G}$ topological groupoid and denoted by $\Gamma \ltimes M \rightrightarrows M$ or by $\left(\Gamma \ltimes M, s_{1}, t_{1}, \mu_{1}, \epsilon_{1}\right.$, inv $\left._{1}, M\right)$.

In the $\mathcal{G}$-topological groupoid $\left(\Gamma \ltimes M, s_{1}, t_{1}, \mu_{1}, \epsilon_{1}\right.$, inv $\left.v_{1}, M\right)$, an arrow from $m$ to $m^{\prime}$ is a pair $(\alpha, m)$ such that the equality $\alpha^{\prime} m=m^{\prime}$. Also we can list the structure maps of the $\mathcal{G}$-topological groupoid $\left(\Gamma \ltimes M, s_{1}, t_{1}, \mu_{1}, \epsilon_{1}, i n v_{1}, M\right)$ as follows:

$s_{1}(\alpha, m)=m$,

$t_{1}(\alpha, m)=\alpha^{\cdot} m=m^{\prime}$

$\epsilon_{1}(m)=(\epsilon(J(m)), m)$,

$\mu_{1}\left(\left(\beta, m^{\prime}\right),(\alpha, m)\right)=(\mu(\beta, \alpha), m)$ with $m^{\prime}=\alpha^{\prime} m$,

inv $_{1}(\alpha, m)=\left(\alpha^{-1}, \alpha^{\prime} m\right)$, where $\alpha^{-1}$ is the inverse element of $\alpha \in \Gamma$.

Now let us show that the $\mathcal{G}$-continuities of the structure maps. $\mathcal{G}$-continuity of the source map $s_{1}$ follows from the $\mathcal{G}$-continuity of the second projection. The target map $t_{1}$ is itself $\mathcal{G}$-action, so it is obvious $\mathcal{G}$-continuous. The object map $\epsilon_{1}$ given by the product $\epsilon \times I d:$ $M \rightarrow \Gamma \ltimes M$ of the identity Id and the object map $\epsilon$ of $\mathcal{G}$-topological groupoid $\left(\Gamma, \Gamma_{0}\right)$ is $\mathcal{G}$-continuous. Also the inverse map inv $v_{1}$ is $\mathcal{G}$-continuous, because inv $v_{1}$ is defined as the product of the inverse map inv and the $\mathcal{G}$-action. Finally, the partial multiplication can be considered as a composition as follows:

$$
\begin{gathered}
(\Gamma \ltimes M)_{s_{1}^{\times} t_{1}}(\Gamma \ltimes M) \stackrel{C_{2,3}}{\longrightarrow}(\Gamma \times \Gamma) \times(M \times M) \stackrel{\mu \times I d}{\longrightarrow} \Gamma \times M \times M \stackrel{\left(\pi_{1} \times \pi_{3}\right)}{\longrightarrow}(\Gamma \ltimes M) \\
((\beta, n),(\alpha, m)) \longmapsto((\beta, \alpha),(n, m)) \longmapsto(\mu(\beta, \alpha), n, m) \longmapsto(\mu(\beta, \alpha), m)
\end{gathered}
$$

, where $\pi_{1}$ is the first projection, $\pi_{3}$ is the third projection and $C_{2,3}$ is the twisting function that changes the locations of the second and third elements. Since all of these maps are $\mathcal{G}$-continuous, the partial multiplication $\mu_{1}$ is $\mathcal{G}$-continuous.

Consequently, $\left(\Gamma \ltimes M, s_{1}, t_{1}, \mu_{1}, \epsilon_{1}\right.$, inv $\left.v_{1}, M\right)$ is a $\mathcal{G}$-topological groupoid.

The following definition gives a notion of action of a $\mathcal{G}$-topological groupoid on another $\mathcal{G}$-topological groupoid.

Definition 3.17. Let $\left(\Gamma, \Gamma_{0}\right)$ and $\left(\Omega, \Omega_{0}\right)$ be $\mathcal{G}$-topological groupoids and let $J: \Omega \rightarrow$ $\Gamma_{0}$ be a $\mathcal{G}$-continuous map, where $\Gamma_{0}$ is regarded as a $\mathcal{G}$-topological groupoid consist of identities only. Then it is called that $\Gamma$ acts on $\Omega$ via $J$ if for each $\alpha \in \Gamma(m, n)$ and each element $\beta \in J^{-1}[\mathrm{~m}]$ there is an arrow $\alpha \beta \in J^{-1}[n]$ such that the following rules hold:

i) $1_{J(\beta)} \cdot \beta=\beta, \forall \beta \in \Omega$

ii) $\alpha \cdot\left(\beta_{2} \bullet \beta_{1}\right)=\left(\alpha \cdot \beta_{2}\right) \bullet\left(\alpha \cdot \beta_{1}\right), \forall \beta_{1}, \beta_{2} \in \Omega, \forall \alpha \in \Gamma$

iii) $\alpha_{1}^{\cdot}\left(\alpha_{2}^{\cdot} \beta\right)=\left(\alpha_{1} \circ \alpha_{2}\right)^{\cdot} \beta, \forall \alpha_{1}, \alpha_{2} \in \Gamma, \beta \in \Omega$.

In that case it is said that $\Omega$ is a $\mathcal{G}$ - $\Gamma$-groupoid.

If $\Omega$ is discrete, this definition be same with the Definition 3.13 , where by a discrete groupoid we mean a groupoid that has only unit arrows as morphisms. 
Definition 3.18. Let $\Gamma$ be a $\mathcal{G}$-topological groupoid acting on both $\mathcal{G}$-topological spaces $M$ and $N$. A $\mathcal{G}$-continuous map $\varphi: M \rightarrow N$ is $\Gamma$-equivariant iff $J_{M}(m)=J_{N}(\varphi(m))$ and $\varphi\left(\alpha^{\prime} m\right)=\alpha^{\cdot} \varphi(m), \forall m \in M$, where $s(\alpha)=J(m)$.

The conditions in the definition are equivalent to being that the following diagrams are commutative:
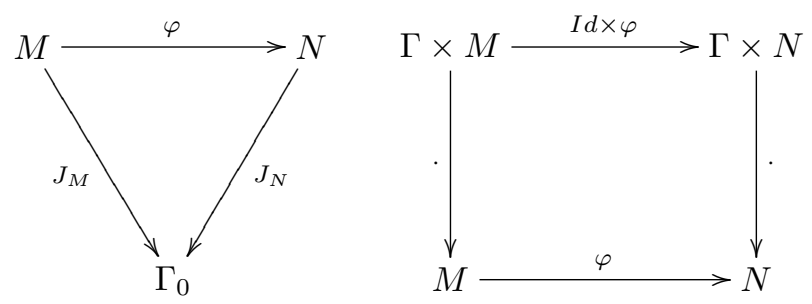

More generally, we can generalize the definition above for two $\mathcal{G}$-topological groupoids in the following way.

Let $\Gamma$ and $\Gamma^{\prime}$ be $\mathcal{G}$-topological groupoids acting on $\mathcal{G}$-topological spaces $M$ and $M^{\prime}$, respectively. Let $\lambda: \Gamma \rightarrow \Gamma^{\prime}$ be a $\mathcal{G}$-homomorphism, let $\varphi: M \rightarrow M^{\prime}$ be a $\mathcal{G}$-continuous map. In this case, if the diagram

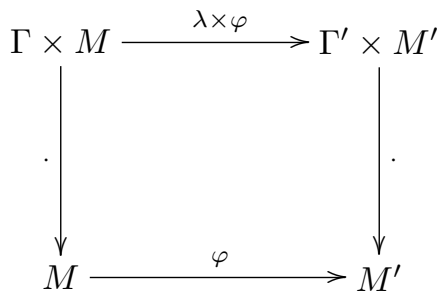

is commutative, then $\varphi$ is called equivariant map.

An action of a $\mathcal{G}$-topological groupoid on a GTS defines a relation as follows:

Definition 3.19. Let $\left(\Gamma, \Gamma_{0}\right)$ be a $\mathcal{G}$-topological groupoid acting on a GTS $M$. We define a relation, called orbit relation, on $M$ as follows:

$$
m \sim n \text { iff } \exists \alpha \in \Gamma \text { such that } \alpha^{\prime} m=n
$$

We denote the orbit (or quotient) space according to this relation by $M / \Gamma$. Its elements are said the orbits of the $\mathcal{G}$-action and are denoted by $\Gamma \cdot m$, for $m \in M$. Also, the canonical projection which assigns to each $m$ in $M$ its orbit, is (often) denoted by $\pi$. Furthermore, $M / \Gamma$ has quotient $\mathcal{G}$-topology by the canonical projection $\pi: M \rightarrow M / \Gamma$ (namely, a set $O \subset M / \Gamma$ is $\mathcal{G}$-open in $M / \Gamma$ iff the $\pi^{-1}(O)$ is $\mathcal{G}$-open in $\left.M\right)$. The $G T S M / \Gamma$ is called the orbit space.

Proposition 3.20. The orbit relation defined above is an equivalence relation.

Proof. The relation $\sim$ is reflexive, because from the third conditions of $\mathcal{G}$-action we have $1_{m} m=m$. For any $m, n \in M$, let $m \sim n$. Hence there is an arrow $\alpha \in \Gamma$ such that $n=\alpha^{\prime} m$. Since there exists $\alpha^{-1} \in \Gamma$ such that $\alpha^{-1} n=m$, it follows that $n \sim m$. Finally if $m \sim n$ and $n \sim k$ then there exist arrows $\alpha, \beta \in \Gamma$ such that $n=\alpha m$ and 
$k=\beta \cdot n$. From the second condition of $\mathcal{G}$-action, we have $k=(\beta \circ \alpha) \cdot m$ and $m \sim k$. Thus $\sim$ is an equivalence relation.

\section{4. $\mathcal{G}$-Topological Crossed Modules}

We here introduce a new concept with named of $\mathcal{G}$-topological crossed module by defining a crossed module over $\mathcal{G}$-topological groupoids. Therefore, we construct the category of $\mathcal{G}$-topological crossed modules.

Definition 4.1. A crossed module over $\mathcal{G}$-topological groupoids is a triplet $\mathcal{C}=(\Upsilon, \Gamma, \eta)$ together with a (left) $\mathcal{G}$-action of $\Gamma$ on $\Upsilon$ and a $\mathcal{G}$-homomorphism $\eta: \Upsilon \rightarrow \Gamma$ (is called boundary map), where the $\mathcal{G}$-topological groupoids $\Upsilon$ and $\Gamma$ have the same object space and $\Upsilon$ is totally intransitive, such that the following conditions hold:

GCR1) $\eta(\alpha \cdot \omega)=\alpha \circ \eta(\omega) \circ \alpha^{-1}$ for $\omega \in \Upsilon(m, m), \alpha \in \Gamma(m, n)$

GCR2) $\eta\left(\omega_{1}\right) \cdot \omega=\omega_{1} \circ \omega \circ \omega_{1}^{-1}$ for $\omega, \omega_{1} \in \Upsilon(m, m)$.

We will say $\mathcal{G}$-topological crossed module for a crossed module of $\mathcal{G}$ - topological groupoids.

Let us now give a main example about $\mathcal{G}$-topological crossed module.

Example 4.2. Let $\Gamma$ be a $\mathcal{G}$-topological groupoid and let $I \Gamma=\bigcup_{m \in \Gamma_{0}} \Gamma(m, m)$ be inner $\mathcal{G}$-topological subgroupoid of $\Gamma$. Then, if we take the inclusion map $i: I \Gamma \rightarrow \Gamma$ as the boundary map $\eta$, we obtain a $\mathcal{G}$-topological crossed module $\mathcal{C}=(I \Gamma, \Gamma, i)$.

Firstly, let us define the $\mathcal{G}$-action of $\Gamma$ on $I \Gamma$. The $\mathcal{G}$-topological groupoid $\Gamma$ acts on inner $\mathcal{G}$-topological subgroupoid $I \Gamma$ via the moment map $J: I \Gamma \rightarrow \Gamma_{0}, J(\omega)=s(\omega)=m$ for any $\omega \in \Gamma(m, m)$ as follows by the partial multiplication of $\Gamma$ :

$$
: \Gamma \times I \Gamma \rightarrow I \Gamma,(\alpha, \omega) \mapsto \alpha^{\cdot} \omega=\alpha^{-1} \circ \omega \circ \alpha .
$$

Let us now control the rules in Definition 3.17 are hold.

i) Let us fixed any $\omega \in I \Gamma$ as $\omega \in \Gamma(m, m) \subset I \Gamma$. Then

$$
1_{J(\omega)} \omega=1_{m}^{\cdot} \omega=1_{m} \circ \omega \circ 1_{m}^{-1}=1_{m} \circ \omega \circ 1_{m}=\omega .
$$

ii) For any elements $\omega_{1}, \omega_{2} \in \Gamma(m, m) \subset I \Gamma$ and $\alpha \in \Gamma(m, n)$,

$$
\begin{aligned}
\alpha^{\cdot}\left(\omega_{1} \circ \omega_{2}\right) & =\alpha \circ\left(\omega_{1} \circ \omega_{2}\right) \circ \alpha^{-1}=\alpha \circ \omega_{1} \circ 1_{m} \circ \omega_{2} \circ \alpha^{-1} \\
& =\alpha \circ \omega_{1} \circ \alpha^{-1} \circ \alpha \circ \omega_{2} \circ \alpha^{-1} \\
& =\alpha \omega_{1} \circ \alpha \omega_{2} .
\end{aligned}
$$

iii) For any $\alpha_{1} \in \Gamma(m, n), \alpha_{2} \in \Gamma(n, k)$ and $\omega \in \Gamma(m, m) \subset I \Gamma$,

$$
\begin{aligned}
\left(\alpha_{1} \circ \alpha_{2}\right)^{\cdot} \omega & =\left(\alpha_{1} \circ \alpha_{2}\right) \circ \omega \circ\left(\alpha_{1} \circ \alpha_{2}\right)^{-1}=\alpha_{1} \circ \alpha_{2} \circ \omega \circ \alpha_{2}^{-1} \circ \alpha_{1}^{-1} \\
& =\alpha_{1} \circ\left(\dot{\alpha_{2}} \omega\right) \circ \alpha_{1}^{-1} \\
& =\alpha_{1}\left(\dot{\alpha_{2}} \omega\right) .
\end{aligned}
$$

On the other hand, it is obvious that the action is $\mathcal{G}$-continuous, since it is defined by the partial multiplication of $\mathcal{G}$-topological groupoid $\Gamma$. Therefore, $\Gamma$ acts on $I \Gamma$ via J.

Now let us show that the conditions of (GCR1) and (GCR2) are hold. 
GCR1)

$$
\begin{aligned}
\eta\left(\alpha^{\prime} \omega\right) & =\eta\left(\alpha \circ \omega \circ \alpha^{-1}\right)=i\left(\alpha \circ \omega \circ \alpha^{-1}\right)=\alpha \circ \omega \circ \alpha^{-1}=\alpha \circ i(\omega) \circ \alpha^{-1} \\
& =\alpha \circ \eta(\omega) \circ \alpha^{-1} .
\end{aligned}
$$

GCR2)

$$
\eta\left(\omega_{1}\right)^{\cdot} \omega=\eta\left(\omega_{1}\right) \circ \omega \circ \eta\left(\omega_{1}\right)^{-1}=i\left(\omega_{1}\right) \circ \omega \circ i\left(\omega_{1}\right)^{-1}=\omega_{1} \circ \omega \circ \omega_{1}^{-1} .
$$

Therefore, $\mathcal{C}=(I \Gamma, \Gamma, i)$ is a $\mathcal{G}$-topological crossed module.

Definition 4.3. Let $\mathcal{C}=(\Upsilon, \Gamma, \eta)$ and $\mathcal{C}^{\prime}=\left(\Upsilon^{\prime}, \Gamma^{\prime}, \eta^{\prime}\right)$ be $\mathcal{G}$-topological crossed modules. A pair $f=\left(f_{1}, f_{2}\right): \mathcal{C} \rightarrow \mathcal{C}^{\prime}$ is called a homomorphism of $\mathcal{G}$-topological crossed modules, if the $\mathcal{G}$-continuous maps $f_{1}: \Gamma \rightarrow \Gamma^{\prime}$ and $f_{2}: \Upsilon \rightarrow \Upsilon^{\prime}$ hold $f_{1} \eta=\eta^{\prime} f_{2}$ and $f_{2}(\alpha \cdot \omega)=$ $f_{1}(\alpha) \cdot f_{2}(\omega)$.

The conditions in the definition are equivalent to be commutative of the following diagrams:
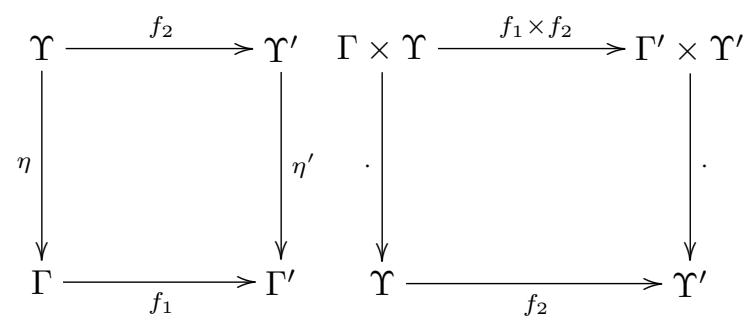

Therefore, $\mathcal{G}$-topological crossed modules form a category denoted by $\mathcal{G} \mathcal{T} C r M$.

\section{From $\mathcal{G} \mathcal{T} G p d$ To $\mathcal{G} \mathcal{T} C r M$ AND FROM $\mathcal{G} \mathcal{T} C r M$ TO $\mathcal{G} \mathcal{T} G p d$}

In the last part of the study, we prove how a generalized topological crossed module can be obtained when given a generalized topological groupoid, and how a generalized topological groupoid can be obtained from it when given a generalized topological crossed module.

Theorem 5.1. Every $\mathcal{G}$-topological groupoid $\left(\Gamma, \Gamma_{0}\right)$ induces a $\mathcal{G}$-topological crossed module.

Proof. To show that a $\mathcal{G}$-topological groupoid $\left(\Gamma, \Gamma_{0}\right)$ induces a $\mathcal{G}$-topological crossed module $\mathcal{C}=(\Upsilon, \Gamma, \eta)$, we need a totally intransitive $\mathcal{G}$ - topological groupoid $\Upsilon$ and a $\mathcal{G}$-topological groupoid that acts on $\Upsilon$, both of which have the same object space. Furthermore, we must show that $\delta: \Upsilon \rightarrow \Gamma$ is a $\mathcal{G}$-homomorphism.

If we consider the isotropy groups $\Upsilon(m)=\{\alpha \in \Gamma \mid s(\alpha)=t(\alpha)=m\}, m \in \Gamma_{0}$, which each is a $\mathcal{G}$-topological group, then we obtain the $\mathcal{G}$-topological groupoid $\bigcup_{m \in \Gamma_{0}} \Upsilon(m)$ that is totally intransitive. We denote it as $\Upsilon=\bigcup_{m \in \Gamma_{0}} \Upsilon(m)$.

From Example 4.2, we have the $\mathcal{G}$-action of $\mathcal{G}$-topological groupoid $\Gamma$ on the totally intransitive $\mathcal{G}$-topological groupoid $\Upsilon=\bigcup_{m \in \Gamma_{0}} \Upsilon(m)$. 
On the other hand, we determine the boundary map $\eta$ as inclusion map $\eta=i: \Upsilon=$ $\bigcup_{m \in \Gamma_{0}} \Upsilon(m) \rightarrow \Gamma$

After these determinations, let us control the conditions GCR1) and GCR2). GCR1)

$$
\begin{aligned}
\eta(\alpha \cdot \omega) & =\eta\left(\alpha \circ \omega \circ \alpha^{-1}\right)=i\left(\alpha \circ \omega \circ \alpha^{-1}\right)=\alpha \circ \omega \circ \alpha^{-1}=\alpha \circ i(\omega) \circ \alpha^{-1} \\
& =\alpha \circ \eta(c) \circ \alpha^{-1} .
\end{aligned}
$$

GCR2)

$$
\eta\left(\omega_{1}\right) \cdot \omega=\eta\left(\omega_{1}\right) \circ \omega \circ \eta\left(\omega_{1}\right)^{-1}=i\left(\omega_{1}\right) \circ \omega \circ i\left(\omega_{1}\right)^{-1}=\omega_{1} \circ \omega \circ \omega_{1}^{-1} .
$$

Therefore $\mathcal{C}=(\Upsilon, \Gamma, i)$ is a $\mathcal{G}$-topological crossed module. Consequently, each $\mathcal{G}$ topological groupoid $\left(\Gamma, \Gamma_{0}\right)$ contains a $\mathcal{G}$-topological crossed module.

As a result of this theorem, we define a functor $\Theta: \mathcal{G} T G p d \rightarrow \mathcal{G T} C r M$.

Theorem 5.2. Every $\mathcal{G}$-topological crossed module $\mathcal{C}=(\Upsilon, \Gamma, \eta)$ induces a $\mathcal{G}$-topological groupoid $\Gamma^{\prime}$.

Proof. Let $\mathcal{C}=(\Upsilon, \Gamma, \eta)$ be a $\mathcal{G}$-topological crossed module. Then, we can obtain the action $\mathcal{G}$-topological groupoid using the $\mathcal{G}$-action of $\Gamma$ on $\Upsilon$. This is the main idea of proof.

Let us consider the product $\mathcal{G}$-topological space $\Gamma^{\prime}=\Gamma \ltimes \Upsilon=\{(\alpha, \omega) \mid \alpha \in \Gamma, \omega \in$ $\Upsilon(t(\alpha))\}$. Then, the algebraic and $\mathcal{G}$-topological details of $\Gamma^{\prime}=\Gamma \ltimes \Upsilon$ are obtained in the same way as in Example 3.16 (see Example 3.16).

As a result of this theorem, we define a functor $\Sigma: \mathcal{G T C r} M \rightarrow \mathcal{G T G p d}$.

Remark 5.3. It can normally be expected that these categories should be equivalent. But the example given below is a counterexample showing that this is not true.

Example 5.4. We know from the Example 3.3 that every Haussdorf $\mathcal{G}$-topological group $\Gamma$ is a $\mathcal{G}$-topological groupoid with one object over its identity, namely $\Gamma \rightrightarrows *$. According to the Theorem 5.1, we obtain a $\mathcal{G}$-topological crossed module $\Theta(\Gamma)=(\Upsilon=\Gamma, \Gamma, i)$, where $\Gamma$ acts on itself via the conjugation. Now let us apply the functor $\Sigma$ to $\Theta(\Gamma)=$ $(\Gamma, \Gamma, i)$. Then, according to the Theorem 5.2, we obtain an action $\mathcal{G}$-topological groupoid $\Sigma \circ \Theta(\Gamma)=\Gamma \times \Gamma$ over $\Gamma$ with $s(\alpha, \beta)=\beta$ and $t(\alpha, \beta)=\alpha \beta \alpha^{-1}$, where $\Gamma$ acts on $\Gamma$ by the conjugation.

It is clear that the $\mathcal{G}$-topological groupoids $\Gamma \rightrightarrows *$ and $\Gamma \times \Gamma \rightrightarrows \Gamma$ are not isomorphic in the category $\mathcal{G} T G p d$, because their object spaces are different. Therefore, the functor $\Sigma \circ \Theta$ is not naturally equivalent to the functor $1_{\mathcal{G T G} \text {. }}$.

\section{ACKNOWLEDGMENTS}

I would like to thank to the unknown referees for their expert suggestions to improve this article. 


\section{REFERENCES}

[1] S.Bayhan, A.Kanibir, I.L.Reilly, On functions between generalized topological spaces, Appl. Gen. Topol. 14 No.2 (2013) 195-203.

[2] H.Brandt, Uber eine Verallgemeinerung des Gruppenbegriffes, Math. Ann. 96, (1926) 360-366.

[3] R.Brown, Topology and Groupoids BookSurge LLC, Deganwy, United Kingdom, 2006.

[4] R.Brown, P.J.Higgins, The Algebra of Cubes, J. Pure Appl. Algebra 21, (1981) 233-260.

[5] R.Brown, I.Icen, Homotopies and automorphisms of crossed module over groupoids, Appl. Categ. Structures 11, (2003) 185-206.

[6] Á.Császár, Generalized topology, generalized continuity, Acta Math. Hungar. 96, No.4 (2002) 351-357.

[7] Á.Császár, Product of generalized topologies, Acta Math. Hungar. 123, No.1-2 (2009) 127-132.

[8] Á.Császár, Separation axioms for generalized topologies, Acta Math. Hungar. 104, No. 1-2 (2004) 63-69.

[9] C.Ehresmann, Catégories topologiques et catégories differentiables, Colloque de géometrie différentielle globale Bruxelles, C.B.R.M, (1958) 137-150.

[10] S.A.Ghour, A.A.Omari, T.Noiri, On homogeneity and homogeneity components in generalized topological spaces, Filomat 27, No. 6 (2013) 1097-1105.

[11] M.H.Gursoy, Actions of vector groupoids, Bull. Iranian Math. Soc. 40, No.3 (2014) 565-582.

[12] M.Hussain, M.D.Khan, C.Ozel, On generalized topological groups, Filomat 27, No. 4 (2013) 567-575.

[13] M.Hussain, M.D. Khan, A.Z.Ozcelik, C.Ozel, Extension closed properties on generalized topological groups, Arab. J. Math. 3, (2014) 341-347.

[14] V.Pankajam, D.Sivaraj, Some separation axioms in generalized topological spaces, Bol. Soc. Parana. Mat. 31, No. 1 (2013) 29-42.

[15] T.Porter, Extensions, crossed modules and internal categories in categories of groups with operations, Proc. Edinb. Math. Soc. 30, (1987) 371-381.

[16] L.E.A.Saraiva, Generalized quotient topologies, Acta Math. Hungar. 132, No.1-2 (2011) 168-173.

[17] D.S.Zhong, L.G.He, On the Action of a Groupoid on a Manifold, Acta Math. Sin. (Engl. Ser.) 19, No. 4 (2003) 745-756. 